\title{
Clinical utility gene card for: acrodermatitis enteropathica
}

\author{
Sébastien Küry ${ }^{\star}, 1$ Monia Kharfi ${ }^{2}$, Sébastien Schmitt $^{1}$ and Stéphane Bézieau ${ }^{1}$ \\ European Journal of Human Genetics (2012) 20, doi:10.1038/ejhg.2011.227; published online 14 December 2011
}

\section{DISEASE CHARACTERISTICS}

1.1 Name of the disease (synonyms)

Acrodermatitis enteropathica (AEZ).

\subsection{OMIM\# of the disease}

201100.

1.3 Name of the analysed genes or DNA/chromosome segments SLC39A4. ${ }^{1}$

\subsection{OMIM\# of the gene(s)}

607059 .

\subsection{Mutational spectrum}

AEZ is inherited as an autosomal recessive trait; as expected, the vast majority of mutations are found in a homozygous or compound heterozygous state. A total of 29 mutations have been reported so far: 15 missense, 3 nonsense, 3 splice site and 8 frameshift mutations. ${ }^{2-6}$ Among seven other variants of uncertain pathogenicity also reported, ${ }^{2}$ three ones, including two missense and a splice site variant, can be considered likely deleterious. No hotspot mutation region is observed, because alterations are evenly distributed all along the 12 exons.

\subsection{Analytical methods}

Bi-directional sequencing of the 12 exons and their flanking intronic sequences is used for the detection of point mutations and small rearrangements. QMPSF (quantitative multiplex PCR of short fluorescent fragments) is used for the screening of large rearrangements involving one or more exons.

\subsection{Analytical validation}

The first level of validation is the sequencing of both strands. Any suspected new mutation present on both strands is then submitted to an internal validation through analysis of mutations and polymorphisms found in previous patients; it is also compared with public databases (eg, dbSNP) and its potential pathogenic effect is searched through prediction bioinformatic programmes. The segregation between the variant and the phenotype is assessed by testing affected/unaffected relatives and obligatory carriers in the family, to avoid possible polymorphisms.
1.8 Estimated frequency of the disease (incidence at birth ('birth prevalence') or population prevalence) The only data available in the literature indicates an incidence of 1:500 000 births in Denmark ${ }^{7}$ (http://www.orpha.net/orphacom/ cahiers/docs/GB/Prevalence_of_rare_diseases_by_alphabetical_list.pdf), which is, however, very hard to verify. An unofficial study conducted in France in the late 90s identified 15 families. The frequency seems much higher in countries of the Mediterranean basin, probably because of a founder effect due to a higher incidence of consanguinity.

1.9 If applicable, prevalence in the ethnic group of investigated person

Not applicable.

\subsection{Diagnostic setting}

\begin{tabular}{lcc}
\hline & Yes & No \\
\hline A. (Differential) diagnostics & $\bigotimes$ & $\square$ \\
B. Predictive testing & $\square$ & $\square$ \\
C. Risk assessment in relatives & $\bigotimes$ & $\square$ \\
D. Prenatal & $\square$ & $\square$ \\
\hline
\end{tabular}

Comment:

AEZ is an autosomal recessive syndrome of severe zinc deficiency due to a malabsorption of zinc by a defective zinc transporter SLC39A4, expressed mainly in the brush border of jejunum and duodenum enterocytes. ${ }^{1}$ (Differential) diagnostics - testing of SLC39A4 is indicated in patients who developed clinical symptoms and biological signs of a severe zinc deficiency, at birth or after weaning (clinical and biological features are described in section 3.1.2). The identification of a molecular anomaly in SLC39A4 gives definitive proof of AEZ and enables its distinction from acquired zinc deficiency, which can be due to either total parenteral nutrition, prematurity or to reduced milk zinc concentration in nursing mothers of exclusively breast-fed infants. In rarer cases, genetic testing may also differentiate AEZ from biotin deficiency or atopic dermatitis, especially when biochemical results are ambiguous (see 3.1.3). Predictive testing - it is not applicable in most cases, because AEZ symptoms and signs occur in the perinatal period. The value of the genetic test would therefore rather be considered diagnostic than predictive. Prenatal - it is technically feasible, but it is not recommended; indeed, the risk for

${ }^{1} \mathrm{CHU}$ Nantes, Institut de Biologie, Laboratoire de génétique moléculaire, Service de Génétique Médicale, Nantes, France; ${ }^{2}$ Hôpital Charles Nicolle, Service de Dermatologie, Tunis, Tunisia

*Correspondence: Dr S Küry, CHU de Nantes Hôtel-Dieu, Institut de Biologie, Service de Génétique Médicale, Laboratoire de génétique moléculaire, 9 quai Moncousu, 44093 Nantes cedex 1, France. Tel: +33 2400842 99; Fax: +33 2400876 47; E-mail: sebastien.kury@chu-nantes.fr

Received 14 September 2011; revised 21 October 2011; accepted 11 November 2011 
the mother inherent to this testing cannot be neglected, whereas, on the other hand, zinc supplementation can be applied immediately in a newborn suspected of AEZ on the basis of a zinc dosage (see 3.1.2.). Genetic testing comes in a second time, in order to confirm the clinical diagnosis.

\section{TEST CHARACTERISTICS}

\begin{tabular}{|c|c|c|c|c|}
\hline & \multicolumn{2}{|c|}{ Genotype or disease } & \multirow{2}{*}{$\begin{array}{l}\text { A: True positives } \\
\text { B: False positives }\end{array}$} & \multirow{2}{*}{$\begin{array}{l}\text { C: False negative } \\
\text { D: True negative }\end{array}$} \\
\hline & Present & Absent & & \\
\hline \multicolumn{5}{|l|}{ Test } \\
\hline Positive & A & B & $\begin{array}{l}\text { Sensitivity: } \\
\text { Specificity: }\end{array}$ & $\begin{array}{l}A /(A+C) \\
D /(D+B)\end{array}$ \\
\hline Negative & C & $\mathrm{D}$ & $\begin{array}{l}\text { Positive predictive value: } \\
\text { Negative predictive value: }\end{array}$ & $\begin{array}{l}A /(A+B) \\
D /(C+D)\end{array}$ \\
\hline
\end{tabular}

\subsection{Analytical sensitivity}

(proportion of positive tests if the genotype is present)

Theoretically $100 \%$, if mutations are localized within exons or flanking introns sequences, which encompasses at least $80 \%$ of all mutations.

\subsection{Analytical specificity} (proportion of negative tests if the genotype is not present) $100 \%$.

\subsection{Clinical sensitivity}

(proportion of positive tests if the disease is present)

The clinical sensitivity can be dependent on variable factors such as age or family history. In such cases a general statement should be given, even if a quantification can only be made case by case.

Overall, mutations of SLC39A4 are observed in about $48 \%$ of the index cases tested: $\sim 40 \%$ of the patients are homozygotes or compound heterozygotes, whereas $8 \%$ are heterozygotes only. In these last ones, we cannot exclude the presence of a second anomaly in unexplored regions of the SLC39A4 gene (introns, 5'UTR, 3'UTR, upstream regulating regions) or in another gene involved in zinc homeostasis, or even the participation of epigenetic events such as methylation. The same hypotheses are also valuable to explain the failure in mutation detection in about $52 \%$ of the patients. In addition, the difficulty - or even sometimes the impossibility - to distinguish congenital zinc deficiency AEZ from acquired zinc deficiency largely accounts for these negative tests; excepted the age at onset, which can be much later in acquired zinc deficiency, both entities share indeed the same clinical features. A few other negative tests are attributable to the partial clinical overlap between AEZ and biotin deficiency or atopic dermatitis.

\subsection{Clinical specificity}

(proportion of negative tests if the disease is not present)

The clinical specificity can be dependent on variable factors such as age or family history. In such cases a general statement should be given, even if a quantification can only be made case by case. $100 \%$.

\subsection{Positive clinical predictive value} (lifetime risk of developing the disease if the test is positive) If homozygous or compound heterozygous SLC39A4 mutations are identified in a yet asymptomatic breast-fed children - their zinc deficiency is masked by zinc from maternal milk the risk to develop AEZ after weaning is $100 \%$.
2.6 Negative clinical predictive value (probability not to develop the disease if the test is negative) Assume an increased risk based on family history for a non-affected person. Allelic and locus heterogeneity may need to be considered.

Index case in that family had been tested:

When a pathogenic SLC39A4 mutation is identified in the index case, the negative predictive value is $100 \%$.

Index case in that family had not been tested:

Not applicable.

\section{CLINICAL UTILITY}

3.1 (Differential) diagnosis: the tested person is clinically affected (To be answered if in 1.10 'A' was marked)

\subsubsection{Can a diagnosis be made other than through a genetic test?}

\begin{tabular}{|c|c|c|}
\hline No & $\square$ (continue with 3.1 .4 ) & \\
\hline \multirow[t]{7}{*}{ Yes } & $\otimes$ & \\
\hline & Clinically & $\otimes$ \\
\hline & Imaging & $\square$ \\
\hline & Endoscopy & $\square$ \\
\hline & Biochemistry & $\otimes$ \\
\hline & Electrophysiology & $\square$ \\
\hline & Other (please describe) & \\
\hline
\end{tabular}

\subsubsection{Describe the burden of alternative diagnostic methods to the patient}

Diagnosis can be established by the concomitant presence of characteristic clinical features and biological signs. Historically, AEZ was defined by a triad of symptoms, including a periacral and periorificial dermatitis, an alopecia and a diarrhea. ${ }^{8}$ In practical terms, this pathognomonic triad is seen in only $25 \%$ of the cases. ${ }^{9}$ The most constant clinical symptoms are cutaneous lesions (first eczematous, then quickly erosive, psoriasiform or vesiculopustulous) always located at least in peri-orificial areas, and very frequently associated with acral lesions. Secondary fungal and bacterial infections, erosions of the buccal mucosa, integument disorders (alopecia, nail dystrophy), diarrhea, neuropsychiatric symptoms (irritability, apathy or psychomotor delay), and failure to thrive are also frequently reported. ${ }^{9}$ The characteristic biological sign of AEZ is a decrease in body zinc levels, usually assessed by a dosage of zinc plasma level; low levels of zincdependent enzymes, such as alkaline phosphatase, are also observed. Consequently, a key element in the diagnosis of AEZ is the excellent responsiveness of zinc deficiency symptoms and signs to oral zinc supplementation; all of them should indeed resolve within days/weeks. It is worth noting however, that such a responsiveness to zinc therapy is also observed in transient neonatal zinc deficiency (acquired zinc deficiency of lactogenic origin), in which maternal milk does not provide enough zinc to meet the infant's needs, despite a normal intestinal absorption. ${ }^{10}$ Eventually, interruption of zinc therapy may help to distinguish between inherited (AEZ) and acquired zinc deficiency, as, on the one hand, a relapse is always observed in AEZ, whereas, on the other hand, no further supplementation is required after weaning in transient neonatal zinc deficiency.

\subsubsection{How is the cost effectiveness of alternative diagnostic methods to be judged?}

Establishing a diagnosis of AEZ is not very easy, because of the rarity of the disease, which often leads to a long diagnostic delay. Moreover, clinical and biochemical diagnostic methods are rarely sufficient to establish a definitive diagnosis of AEZ, notably because acquired zinc 
deficiency causes are varied and not always obvious to determine. ${ }^{11,12}$ More rarely, biotinidase deficiency or atopic dermatitis are sometimes misdiagnosed as AEZ, especially when zinc levels are found subnormal and symptoms seem zinc responsive. Such situations can be considered as exceptions, but they nevertheless illustrate the difficulty to rely upon zinc dosage and clinical features only, all the more that true AEZ patients also happen to exhibit very marginal decreases in plasma zinc levels. As a matter of fact, zinc homeostasis is so tightly regulated that even the slightest biochemical abnormality can stress a more profound zinc deficiency. Methods for zinc dosage and definition of standard values for zinc levels are not harmonized either between laboratories/ countries, which can induce misinterpretation of biochemical results. In any case, the ultimate diagnostic proof of AEZ is therefore brought by the identification of a molecular anomaly in SLC39A4.

\subsubsection{Will disease management be influenced by the result of a} genetic test?

\section{No $\square$}

Yes $\otimes$

Therapy (please describe)

Prognosis (please describe)

AEZ patients require a lifelong zinc supplementation. Any interruption of the therapy will inevitably induce a relapse. Once AEZ diagnosis is established, the prognosis is excellent, provided zinc supplementation is correctly followed. On the contrary, if untreated, patients would experience a progressive alteration of all physiological functions expressed by a large cohort of symptoms, including neurological, immunological and gastrointestinal disorders; this would lead to a generalized organic failure and to death.

Management (please The result of the genetic test will necessarily influence describe) disease management. Relevance and duration of zinc therapy in a child depend indeed directly on the result of genetic testing. In homozygotes or compound heterozygotes, confirmation of AEZ diagnosis is essential, because an untreated zinc deficiency may be fatal over the medium to long term; these patients should receive a lifelong zinc treatment. On the other hand, patients with no mutation identified probably have an acquired zinc deficiency, which may resolve either spontaneously or once the true cause of the disease is determined and treated. In any case, a regular clinical and biological follow-up of the patients enables to adapt the dose of zinc supplement to possible physiological (eg, adolescence, pregnancy) or pathological (eg, inflammatory states) variations of zinc levels.

3.2 Predictive setting: the tested person is clinically unaffected but carries an increased risk based on family history

(To be answered if in 1.10 'B' was marked)

3.2.1 Will the result of a genetic test influence lifestyle and prevention?

If the test result is positive (please describe):

Not applicable.

If the test result is negative (please describe):

Not applicable.

3.2.2 Which options in view of lifestyle and prevention does a person at risk have if no genetic test has been done (please describe)? Not applicable.

3.3 Genetic risk assessment in family members of a diseased person (To be answered if in 1.10 ' $\mathrm{C}$ ' was marked)
3.3.1 Does the result of a genetic test resolve the genetic situation in that family?

Yes, given the autosomal recessive mode of inheritance, newborns can be evaluated immediately after birth for the disorder.

3.3.2 Can a genetic test in the index patient save genetic or other tests in family members?

No.

3.3.3 Does a positive genetic test result in the index patient enable a predictive test in a family member?

Yes.

3.4 Prenatal diagnosis

(To be answered if in 1.10 'D' was marked)

3.4.1 Does a positive genetic test result in the index patient enable a prenatal diagnosis?

Not applicable.

\section{IF APPLICABLE, FURTHER CONSEQUENCES OF TESTING}

Please assume that the result of a genetic test has no immediate medical consequences. Is there any evidence that a genetic test is nevertheless useful for the patient or his/her relatives? (Please describe).

Results of SLC39A4 genetic testing may have no immediate medical consequence, because zinc supplementation has almost always been proposed to patients before the request for genetic analysis. Yet, in addition to its influence on disease management in homozygotes or compound heterozygotes (see section 3.1.4), the results of SLC39A4 genetic testing may also have consequences for their relatives. Mutation carriers are worth detecting, as heterozygous carriers of a pathogenic mutation would be hypersensitive to zinc deficiency. ${ }^{13}$ Their zinc status should therefore be regularly followed up to prevent possible symptoms of zinc deficiency. Beside, genetic testing of SLC39A4 may avoid zinc oversupplementation in transiently zincdeficient neonates, who do not need any further zinc therapy after weaning. Of note, in transient neonatal zinc deficiency, a genetic anomaly is suspected that would be carried by the mother and not by the infant, as in AEZ. Beyond SLC39A4 screening, future additional genetic testings may therefore be expected for this other form of zinc deficiency, but to date the mutation observed once in the zinc transporter gene $S L C 30 A 2^{10}$ has not been confirmed in other families. This suggests a genetic heterogeneity and the need for further investigations to unravel the whole pathogenic mechanism leading to transient neonatal zinc deficiency.

\section{CONFLICT OF INTEREST}

The authors declare no conflict of interest.

\section{ACKNOWLEDGEMENTS}

This work was supported by the EuroGentest2 (Unit 2: 'Genetic testing as part of health care'), a Coordination Action under FP7 (Grant Agreement number 261469) and the European Society of Human Genetics. SK, SS and SB are partially supported by a donation from the laboratory LABCATAL for their fundamental research on AEZ.

\footnotetext{
1 Küry S, Dréno B, Bézieau S et al: Identification of SLC39A4, a gene involved in acrodermatitis enteropathica. Nat Genet 2002; 31: 239-240.

2 Schmitt S, Küry S, Giraud M, Dréno B, Kharfi M, Bézieau S: An update on mutations of the SLC39A4 gene in acrodermatitis enteropathica. Hum Mutat 2009; 30: 926-933.
} 
3 Vardi A, Anikster Y, Eisenkraft A et al: A new genetic isolate of acrodermatitis enteropathica with a novel mutation. Br J Dermatol 2009; 160: 1346-1348.

4 Li CR, Yan SM, Shen DB et al: One novel homozygous mutation of SLC39A4 gene in a Chinese patient with acrodermatitis enteropathica. Arch Dermatol Res 2010; 302: 315-317.

5 Park CH, Lee MJ, Kim HJ, Lee G, Park JW, Cinn YW: Congenital zinc deficiency from mutations of the SLC39A4 gene as the genetic background of acrodermatitis enteropathica. J Korean Med Sci 2010; 25: 1818-1820.

6 Jung AG, Mathony UA, Behre B et al: Acrodermatitis enteropathica: an uncommon differential diagnosis in childhood - first description of a new sequence variant. J Dtsch Dermatol Ges 2011; 9: 999-1002.

7 Van Wouwe JP: Clinical and laboratory diagnosis of acrodermatitis enteropathica. Eur J Pediatr 1989; 149: 2-8.

8 Danbolt N, Closs K: Acrodermatitis enteropathica. Acta Dermatol Venereol 1942; 23 : 127-169.
9 Kharfi M, El Fekih N, Aounallah-Skhiri $\mathrm{H}$ et al: Acrodermatitis enteropathica: a review of 29 Tunisian cases. Int J Dermatol 2010; 49: 1038-1044.

10 Chowanadisai W, Lonnerdal B, Kelleher SL: Identification of a mutation in SLC30A2 (ZnT-2) in women with low milk zinc concentration that results in transient neonatal zinc deficiency. J Biol Chem 2006; 281: 39699-39707.

11 Perafan-Riveros C, Franca LF, Alves AC, Sanches Jr JA: Acrodermatitis enteropathica: case report and review of the literature. Pediatr Dermatol 2002; 19: 426-431.

12 Maverakis E, Fung MA, Lynch PJ et al: Acrodermatitis enteropathica and an overview of zinc metabolism. J Am Acad Dermatol 2007; 56: 116-124.

13 Dufner-Beattie J, Weaver BP, Geiser J et al: The mouse acrodermatitis enteropathica gene Sic39a4 (Zip4) is essential for early development and heterozygosity causes hypersensitivity to zinc deficiency. Hum Mol Genet 2007; 16: 1391-1399. 